\title{
Scaling Laws of Multiple Antenna Group-Broadcast Channels
}

\author{
Tareq Y. Al-Naffouri, Amir F. Dana, and Babak Hassibi
}

\begin{abstract}
Broadcast (or point to multipoint) communication has attracted a lot of research recently. In this paper, we consider the group broadcast channel where the users' pool is divided into groups, each of which is interested in common information. Such a situation occurs for example in digital audio and video broadcast where the users are divided into various groups according to the shows they are interested in. The paper obtains upper and lower bounds for the sum rate capacity in the large number of users regime and quantifies the effect of spatial correlation on the system capacity. The paper also studies the scaling of the system capacity when the number of users and antennas grow simultaneously. It is shown that in order to achieve a constant rate per user, the number of transmit antennas should scale at least logarithmically in the number of users.
\end{abstract}

Index Terms-MIMO systems, transmit diversity, broadband mobile communication systems, wireless personal communication systems.

\section{INTRODUCTION}

$\mathbf{F}$ UTURE breakthroughs in wireless communications will be mostly driven by applications that require high data rates [1]. While increasing the link budget and/or bandwidth can accommodate this increase in data rate, such a solution would not be economical. A more cost effective solution is to exploit the spacial dimension by employing multiple antennas at the transmitter and receiver. Multiple input multiple output (MIMO) communication has thus been the focus of a lot of research [1], [2], [3] which basically demonstrated that the capacity of a point to point MIMO link increases linearly with the number of transmit and receive antennas (an excellent overview of the research on this problem can be found in [4]).

Research focus has shifted recently to the role of multiple antennas in multiuser systems, especially broadcast scenarios (i.e., point to multipoint communication) as downlink scheduling is the major bottleneck for future broadband wireless networks. The broadcast channel resembles downlink communication in a cellular system, where the base station is to transmit to a group of users. In these and other broadcast scenarios, one is usually interested in 1) quantifying the system capacity or the maximum possible sum rate to all users, 2) quantifying the scaling behavior of the sum rate for large

Manuscript received August 14, 2007; revised February 17, 2008; accepted June 2, 2008. The associate editor coordinating the review of this paper and approving it for publication was I. Lee.

T. Y. Al-Naffouri is with the Electrical Engineering Department, King Fahd University of Petroleum and Minerals, Saudi Arabia (e-mail: naffouri@kfupm.edu.sa). The work of T. Y. Al-Naffouri was supported by Project IN070373, Deanship of Scientific Research at King Fahd University of Petroleum and Minerals.

A. Dana is with Qualcomm Corporation, San Diego, CA

B. Hassibi is with California Institute of Technology (e-mail: hassibi@caltech.edu).

Digital Object Identifier 10.1109/T-WC.2008.070902 number of users, and 3) devising computationally efficient algorithms for capturing most of the sum rate in the large number of users regime. In this paper, we distinguish between two types of broadcast scenarios depending on whether the users are interested in common information or not.

\section{A. Broadcasting to Independent Users}

In this problem, users are interested in independent information. Much work has been devoted to answer the three questions raised above for this problem. The capacity region question was recently settled by a technique similar to writing on dirty paper and hence bearing the name dirty paper coding (DPC). Specifically, [5] and [6] have shown that DPC is capable of achieving the maximum possible sum-rate capacity. Subsequently, [7] showed that DPC is able to achieve any point in the capacity region.

While DPC solves the broadcast problem optimally, it is computationally expensive and requires a great deal of feedback as the transmitter needs perfect channel state information of all users [8]. Thus, there has been increased interest to match the DPC capacity for large number of users using simple techniques. In [9] and [10], Sharif and Hassibi showed that the sum rate capacity behaves like $M \log n \log n$ for large $n$ (where $n$ is the total number of users and $M$ is the number of transmit antennas). They also showed that opportunistic beam forming matches this limit. Other promising techniques for capturing most of the DPC capacity were proposed in [7], [10], [11], [12]. They all rely on multiuser diversity to match the DPC for large number of users. Here, each user experiences a different channel and therefore the transmitter can exploit this diversity and choose the set of users that have the best channel conditions.

\section{B. Broadcasting to Groups of Users}

The broadcast scenario considered above assumes that the various users are interested in independent streams of data. More common is the situation that one group of users would be interested in one stream of data, another group with another stream, and so on. An example where this might occur is digital audio and video broadcast where there is a limited number of shows and users are classified according to the shows they are interested in [13], [14], [15]. Here, similar questions to the (independent user) broadcast problem would be relevant.

To make the discussion more rigorous, assume that there are $n$ users each equipped with a single antenna. The users are partitioned into $K$ groups where each group is interested in the same stream of data. The transmitter, which is equipped with $M$ antennas, is to schedule transmission to these groups 
so as to maximize the sum-rate capacity of the system. If the transmitter had one antenna only, this would be a trivial problem. For in this case, all channels involved would be single input single output. Thus, to transmit to any group of users, one simply needs to take care of the user with the weakest link (i.e., the one with the least channel gain). Such ordering of users, however, is not possible in the multiple antenna case and the problem becomes more challenging.

The group broadcast problem includes the (independent users) broadcast problem as a special case. Specifically, the independent users broadcast is a group broadcast problem in which each group consists of one user only. The other extreme is when all users belong to one group and are all interested in the same information. This is known as a multicast problem and has started to attract some attention recently. In [16], Khitsi et. al. characterized the system capacity for the two user binary multicast problem. When multicasting to more than 2 users or for Gaussian multicast, [16] was only able to obtain upper and lower bounds. In [17], Steinberg and Shamai considered a two user situation with a hybrid of broadcast and multicast in which the two users can have common as well as independent messages.

By examining the techniques used in [16] and [17] and the results arrived at, one can be convinced that finding the exact capacity region for the multicast problem (let alone the more general group broadcast problem) can be quite challenging. As such, several authors have resorted instead to evaluate the capacity asymptotes. In his Masters Thesis [18], Khitsi considered the multicast problem where there is exactly one group of $n$ users interested in a common message transmitted from a base station with $M$ antennas. He showed that for large $n$, the capacity decreases in proportion to $n^{-\frac{1}{M}}$. In a recent paper [19], Jindal and Luo built on the work of Khisti and obtained the scaling order of various techniques when used in a multicast scenario. These techniques include transmit beamforming and group broadcast using spatially white or orthogonal signaling.

In this paper, we consider the multiple group broadcast in the large number of users and antennas regime. In contrast to the scaling analysis of [18] and [19], we don't just obtain order relationships but rather obtain upper and lower bounds that more tightly characterize the system sum-rate capacity.

The paper is organized as follows. In Section III, we present a general theory for the scaling behavior of the minimum of a number of iid random variables. The rest of the paper is divided into two parts. In the first part, we consider the large number of users $(n)$ case and obtain upper bounds (in Section IV) and lower bounds (in Section V). In the second part, we consider the scaling for the large number of antennas $(M)$ regime. We do so for fixed $n$ (Section VI), for $n=\beta M$ (Section VII) and for $n=e^{M}$ (Section VIII). We set the stage, however, by introducing the system model.

\section{System Model AND Formal DEFINITION OF CAPACITY}

\section{A. System Model}

Consider a broadcast channel with a base station equipped with $M$ antennas and $n$ users each equipped with a single receive antenna. The $n$ users are partitioned into $K$ groups each of which is interested in the same stream of data. Specifically, we assume there are $\alpha_{k} n$ users in the $k$ th group $k=1,2, \ldots, K$ where $\sum_{k=1}^{K} \alpha_{i}=1, \alpha_{k}>0$. We also let $\alpha$ denote $\min _{k}\left\{\alpha_{k}\right\}$ since many of our results will depend on this minimum. Moreover, to get more insights, we will often times specialize our findings to the case where $\alpha=\alpha_{k}=\frac{1}{K}$. The received signal at the $i_{k}$ th user is given by

$$
y_{i_{k}}=h_{i_{k}}^{*} s+\nu_{i_{k}}
$$

where $s$ is the transmitted signal and is subject to an average power constraint $E\left[s s^{*}\right] \leq P I$ and $\nu_{i_{k}} \sim C N(0,1)$ is the additive white complex Gaussian noise. The vector $h_{i_{k}}$ is the channel matrix between the base station and user $i_{k}$. The users' channels are independent and identically distributed as $C N\left(0, I_{M}\right)$. At times, we will assume that $h_{i_{k}}$ exhibits some spatial correlation so that $h_{i_{k}} \sim C N(0, R)$ where $R$ is full rank with $\operatorname{Tr}(R)=M$.

\section{B. Group Sum-Capacity Expression}

In this paper we are interested in the sum-capacity of the group broadcast systems. Let $W_{i}$ be the information stream of rate $R_{i}$ intended for users in group $i$ for each $i=1, \ldots, K$. Then $\left(R_{1}, \ldots, R_{K}\right)$ is an achievable rate vector if each user in each group can decode its intended information stream with arbitrary small probability of error using long enough block codes. We define the sum-capacity of the system as the supremum of sum of rates among all achievable rate vectors.

Consider a multicast system (i.e., a group broadcast with only one group). Given a total power budget $P$, the base station can transmit to one user at the following maximum rate

$$
C_{\text {oneuser }}=E \max _{B \geq 0 \operatorname{Tr}(B) \leq P} \log \operatorname{det}\left(1+\|h\|_{B}^{2}\right)
$$

where $\left\|h_{i}\right\|_{B}^{2} \triangleq h_{i}^{*} B h_{i}$ and $B$ is the correlation of the input. Since each user in the group is interested in the same data, we have to appeal to all users, particularly the weakest one, by minimizing the log det over all users, i.e.

$$
C_{\text {single }}=E \max _{B \geq 0 \operatorname{Tr}(B) \leq P} \min _{i} \log \operatorname{det}\left(1+\left\|h_{i}\right\|_{B}^{2}\right)
$$

The above expression is the sum-capacity of a multicast system. However the sum-capacity of group broadcast problems in general is not known. If the transmitter had one antenna only, this would be a trivial problem. For in this case, all channels involved would be single input single output. Thus, to transmit to the $k$ th group of users, one simply needs to take care of the user with the weakest link, i.e. the link for which $\left|h_{i_{k}}\right| \leq\left|h_{j_{k}}\right|$ for all $j$. Such ordering of users, however, is not possible in the multiple antenna case and the problem becomes more challenging. Hence, we resort in this paper to bounding the capacity in the large number of users and antennas regime.

\section{SCALING LAW FOR THE MINIMUM OF A NUMBER OF RANDOM VARIABLES}

Group broadcast is intuitively limited by the worst of otherwise identical users. As such, we present in this section a theorem for finding the minimum of a large number of random variables. To this end, let $x_{1}, x_{2}, \cdots, x_{n}$ be iid nonnegative random variables with pdf $f(x), \operatorname{CDF} F(x)$, and 
characteristic function $\phi(s)$. We would like to find the scaling law of the minimum of these random variables, $x_{\min }(n)=$ $\min \left\{x_{1}, x_{2}, \cdots, x_{n}\right\}$. The CDF of the minimum is given by $F_{\min }(x)=1-(1-F(x))^{n}$. Let's first illustrate the direct method of finding the scaling of the minimum through the following example.

a) Example 1: Beamforming for multicast: In beamforming [10], the transmitter sends $M$ orthonormal beams $\phi_{1}, \phi_{2}, \ldots, \phi_{M}$, and asks each user to feedback the SINR associated with each beam. For example the SINR associated with beam $\phi_{1}$ for user $i$ is given by

$$
\operatorname{SINR}_{i}=\frac{\left|h_{i}^{*} \phi_{1}\right|^{2}}{\frac{1}{\rho}+\sum_{m=2}^{M}\left|h_{i}^{*} \phi_{m}\right|^{2}}
$$

where $\rho=\frac{M}{P}$ is the signal to noise ratio. Since the base station has to appeal to all users in the group, we are constrained by the worst user. For a given beam, the $\mathrm{SINR}_{i}$ 's are iid with CDF [10]

$$
F_{\text {SINR }}(x)=1-\frac{e^{-\frac{x}{\rho}}}{(1+x)^{M-1}} \quad x \geq 0
$$

So the CDF of the minimum of $n$ such SINR's is given by

$$
\begin{aligned}
F_{\min \operatorname{SINR}}(x) & =1-\left(1-F_{\operatorname{SINR}}(x)\right)^{n} \\
& =1-\frac{e^{-\frac{n x}{\rho}}}{(1+x)^{n(M-1)}} \quad x \geq 0
\end{aligned}
$$

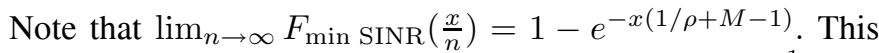
shows that for large $n, \min _{i} \mathrm{SINR}_{i}$ scales as $\frac{1}{\left(\frac{1}{\rho}+M-1\right) n}$. The method of Example 1 might not apply all the time as it is difficult to find the CDF in closed form sometimes. The following theorem provides a more general method for finding the scaling of the minimum. The proof is omitted due to space limitations.

Theorem 1: Let $x_{1}, x_{2}, \cdots, x_{n}$ be iid nonnegative random variables with $\mathrm{CDF} F(x)$, and characteristic function $\phi(s)$. Let $x_{\min }(n)$ denote the minimum of these random variables $\min \left\{x_{1}, x_{2}, \cdots, x_{n}\right\}$. Then $a_{n} x_{\min }(n)$ converges in distribution to a random variable $y$ with $\operatorname{CDF} F_{y}(y)=1-\exp \left(-y^{i_{0}}\right)$ where $i_{0}$ is the first non-zero derivative of $F(x)$ at zero, i.e., $F^{\left(i_{0}\right)}(0) \neq 0$ and $F^{(j)}(0)=0$ for all $j<i_{0}$ and where

$$
a_{n}=\frac{i_{0} !^{\frac{1}{i_{0}}}}{F^{\left(i_{0}\right)}(0)^{\frac{1}{i_{0}}}} \frac{1}{n^{\frac{1}{i_{0}}}}
$$

Furthermore, we can find $F^{\left(i_{0}\right)}(0)$ using the initial value theorem

$$
\lim _{x \rightarrow 0} F^{\left(i_{0}\right)}(x)=\lim _{s \rightarrow \infty} s^{i_{0}} \phi(s)
$$

b) Example 2: Scaling of spatially correlated channel norms: Let's find the scaling law for $\min _{h_{i}}\left\|h_{i}\right\|^{2}$ when $h_{i}$ are iid $C N(0, R)$. The pdf and CDF of $\left\|h_{i}\right\|^{2}$ will both have different forms depending on whether some of the eigenvalues $\lambda_{l}$ of $R$ are the same or different, and so the direct method for scaling can be quite challenging. On the other hand, the characteristic function takes one form and is given by $\phi(s)=$ $\prod_{l=1}^{M} \frac{1}{1+\lambda_{l} s}$. From this, it is easy to see that

$$
\lim _{s \rightarrow \infty} s^{i} \phi(s)=F^{(i)}(0)=\left\{\begin{array}{cc}
0 & \text { for } i<M \\
\frac{1}{\prod_{l=1}^{M} \frac{1}{\lambda_{l}}} & \text { for } i=M
\end{array}\right.
$$

We thus conclude that

$$
\min _{i}\left\|h_{i}\right\|^{2} \text { scales as } C_{M} \operatorname{det}(R)^{\frac{1}{M}} \frac{1}{n^{\frac{1}{M}}}
$$

where $C_{M}=(M !) \frac{1}{M}$.

\section{UPPER BOUNDS}

\section{A. An Upper Bound Using the MAC-BC Duality}

Consider the group broadcast problem with $K$ groups. To obtain an upper bound, we consider all possible broadcast systems with exactly one user chosen from each group. This is a typical broadcast scenario for which the sum-rate capacity is given by dirty paper coding. Using the MAC-BC duality [20], the sum-capacity is given by

$$
C_{K} \text { users }=\max _{\substack{b_{k} \geq 0 \\ \sum b_{k}=P}} \log \operatorname{det}\left(I+\sum_{k=1}^{K} h_{i_{k}} b_{k} h_{i_{k}}^{*}\right)
$$

Now, sweeping over all the possible selections of the K users (one user from each group) we can write

$$
C_{\mathrm{u}}=\min _{h_{i_{1}}} \cdots \min _{h_{i_{K}}} \max _{\substack{b_{k} \geq 0 \\ \sum b_{k}=P}} \log \operatorname{det}\left(I+\sum_{k=1}^{K} h_{i_{k}} b_{k} h_{i_{k}}^{*}\right)
$$

This is an upper bound on the sum-rate because the $b_{k}$ 's are optimized for each set of $K$ users when in the group broadcast problem the $b_{k}$ 's should be the same for all user groups. To get rid of the determinant in (3), we use the arithmetic-mean geometric-mean (AM-GM) inequality

$$
\operatorname{det}(A) \leq\left(\frac{\operatorname{tr}(A)}{M}\right)^{M}
$$

to write

$$
\begin{aligned}
& C_{\mathrm{u}} \leq M \log \left(1+\min _{h_{i_{1}}} \cdots \min _{h_{i_{K}}} \max _{b_{k}} \frac{1}{M} \sum_{k=1}^{K} b_{k}\left\|h_{i_{k}}\right\|^{2}\right) \\
= & M \log \left(1+\frac{P}{M} \min _{h_{i_{1}}} \cdots \min _{h_{i_{K}}} \max _{k}\left\{\left\|h_{i_{1}}\right\|^{2}, \cdots,\left\|h_{i_{K}}\right\|^{2}\right\}\right) \\
= & M \log \left(1+\frac{P}{M} \max _{k} \min _{h_{i_{1}}} \cdots \min _{h_{i_{K}}}\left\{\left\|h_{i_{1}}\right\|^{2}, \cdots,\left\|h_{i_{K}}\right\|^{2}\right\}\right)
\end{aligned}
$$

i.e.

$$
C_{\mathrm{u}} \leq M \log \left(1+\frac{P}{M} C_{M} \max _{k} \frac{1}{\alpha_{k}^{\frac{1}{M}} n^{\frac{1}{M}}}\right)
$$

where the third line follows from exchanging the order of minimum and maximum. Thus, we can write

$$
C_{\mathrm{u}} \leq M \log \left(1+\frac{P}{M} C_{M} \frac{1}{\alpha^{\frac{1}{M}} n^{\frac{1}{M}}}\right)
$$

In the equal number of users case, $\alpha_{k}=\frac{1}{K}$, and the upper bound becomes

$$
C_{\mathrm{u}} \leq M \log \left(1+\frac{P}{M} C_{M} \frac{K^{\frac{1}{M}}}{n^{\frac{1}{M}}}\right)
$$

An alternative upper bound can be obtained as follows. Starting with the MAC-BC inequality (3), let

$$
H_{i}=\left[\begin{array}{lll}
\sqrt{b_{1}} h_{i_{1}} & \cdots & \sqrt{b_{K}} h_{i_{K}}
\end{array}\right]
$$


then, $\sum_{k=1}^{K} h_{i_{k}} b_{k} h_{i_{k}}^{*}=H_{i} H_{i}^{*}$ and the upper bound can be equivalently expressed as

$$
C_{\mathrm{u}}=\min _{h_{i_{1}}} \cdots \min _{h_{i_{K}}} \max _{\substack{b_{k} \geq 0 \\ \sum b_{k}=P}} \log \operatorname{det}\left(I_{K}+H_{i}^{*} H_{i}\right)
$$

Note that the matrix inside the determinant is now of size $K$. So applying the AM-GM inequality this time yields

$$
C_{\mathrm{u}} \leq K \log \left(1+\frac{P}{K} C_{M} \frac{1}{\alpha^{\frac{1}{M}} n^{\frac{1}{M}}}\right)
$$

Combing (8) and (10) yields for the equal number of users case

$$
C_{\mathrm{u}} \leq \min \{M, K\} \log \left(1+\frac{P}{\min \{M, K\}} C_{M} \frac{K^{\frac{1}{M}}}{n^{\frac{1}{M}}}\right)
$$

Using the expansion that for small $x, \log (1+x)=x+\boldsymbol{O}\left(x^{2}\right)$, accurate for small $x$, we can write

$$
C_{\mathrm{u}} \leq P C_{M} \frac{K^{\frac{1}{M}}}{n^{\frac{1}{M}}}+\boldsymbol{O}\left(\frac{1}{n^{\frac{2}{M}}}\right)
$$

In the above, we assumed the $h_{i_{k}}$ 's to be white. In the correlated case, the minimization in (6) is done over $h_{i}$ 's with autocorrelation matrix $R$ and that results in a hit $\operatorname{det}(R)^{\frac{1}{M}}$ on the upper bound ${ }^{1}$

$$
C_{\mathrm{u}, \text { corr }} \leq P C_{M} \operatorname{det}(R)^{\frac{1}{M}} \frac{K^{\frac{1}{M}}}{n^{\frac{1}{M}}}+\boldsymbol{O}\left(\frac{1}{n^{\frac{2}{M}}}\right)
$$

\section{B. How Loose is the Upper Bound?}

The upper bounds obtained above come from two contributions: the BC-MAC duality and the AM-GM inequality. It is thus interesting to see how loose the upper bound becomes due to the use of the AM-GM inequality. To this end, note that for a positive semidefinite matrix $A, \operatorname{det}(I+A) \geq 1+\operatorname{tr}(A)$ which when applied to (3) yields the following lower bound on $C_{\mathrm{u}}$

$$
C_{\mathrm{u}} \geq \min _{h_{i, 1}} \cdots \min _{h_{i, K}} \max _{b_{k}} \log \left(1+\operatorname{tr} \sum_{k=1}^{K} h_{i} b_{k} h_{i}^{*}\right)
$$

Following the steps (4)-(7), we obtain (assuming equal number of users case for simplicity)

$$
C_{\mathrm{u}} \geq \log \left(1+P C_{M} \frac{K^{\frac{1}{M}}}{n^{\frac{1}{M}}}\right)
$$

or using the $\log (1+x)=x+\boldsymbol{O}\left(x^{2}\right)$ approximation

$$
C_{\mathrm{u}} \geq P C_{M} \frac{K^{\frac{1}{M}}}{n^{\frac{1}{M}}}+\boldsymbol{O}\left(\frac{1}{n^{\frac{2}{M}}}\right)
$$

which coincides with (12). So we don't loose anything by applying the AM-GM inequality for large number of users and we thus conclude

$$
C_{\mathrm{u}}=P C_{M} \frac{K \frac{1}{M}}{n^{\frac{1}{M}}}+\boldsymbol{O}\left(\frac{1}{n^{\frac{2}{M}}}\right)
$$

${ }^{1}$ By the AM-GM inequality, we know that $\operatorname{det}(R)^{\frac{1}{M}} \leq \frac{1}{M} \operatorname{tr}(R)=1$.

\section{LOWER BOUNDS}

Having obtained an upper bound on the sum-rate, we now quantify how various methods for scheduling (or resource allocation) behave for large number of users. This gives us an idea about the achievable rates and also provides lower bounds on the group broadcast problem. Specifically, we consider the following scheduling schemes 1) Opportunistic random beamforming 2) Time sharing and 3) Scheduling by treating interference as noise

\section{A. Opportunistic Random Beamforming}

Opportunistic beamforming is a broadcast technique proposed in [9] that captures most of the capacity guaranteed by DPC for large number of users while requiring much less feedback and complexity. Here, the transmitter attempts to choose the best $M$ out of $K$ users to transmit to. To do so, the transmitter uses its $M$ antennas to send $M$ random beams. Each user calculates the $M$ SINR's (signal to interference and noise ratio), one SINR for each beam, and feeds back the maximum SINR along with its index. The transmitter in turn ranks the $K$ users according to their SINR's and transmits to the $M$ best ones. Not only does this method require much less feedback than the DPC approach, but it also asymptotically (i.e., in the presence of large number of users) achieves the same performance [9].

To be more specific, the transmitter chooses $M$ random orthonormal beam vectors $\phi_{m}$ (of size $M \times 1$ ) generated according to an isotropic distribution. Now these beams are used to transmit the symbols $s_{1}(t), s_{2}(t), \ldots, s_{M}(t)$ by constructing the transmit vector

$$
s(t)=\sum_{m=1}^{M} \phi_{m}(t) s_{m}(t), \quad t=1, \ldots, T
$$

After $T$ channel uses, the transmitter independently chooses another set of orthonormal vectors $\left\{\phi_{m}\right\}$ and reconstructs the signal vector $s(t)$ again (according to (18)) and so on. From now on and for simplicity, we will drop the time index $t$. The signal $y_{k}$ received by the $k$ 'th user is given by

$$
\begin{aligned}
y_{k} & =h_{k}^{*} s+n_{k} \\
& =\sum_{m=1}^{M} h_{k}^{*} \phi_{m} s_{m}+n_{k}, \quad k=1, \ldots, K
\end{aligned}
$$

Here $E\left(s s^{*}\right)=P I$ since the $s_{m}$ 's are assumed to be identically distributed and independently assigned to different users. The $k$ 'th receiver estimates the effective channel gain $h_{k}^{*} \phi_{m}$, something that can be arranged by training, to calculate $M$ SINR's, one for each transmitted beam

$$
\mathrm{SINR}_{k, m}=\frac{\left|h_{k} \phi_{m}\right|^{2}}{\frac{1}{\rho}+\sum_{j \neq m}\left|h_{k} \phi_{j}\right|^{2}} \quad m=1, \ldots, M
$$

where $\rho=\frac{M}{P}$ is the signal to noise ratio. Each receiver then feeds back its maximum SINR, i.e. $\max _{1 \leq m \leq M} \mathrm{SINR}_{k, m}$, along with the maximizing index $m$. Thereafter, the transmitter assigns $s_{m}$ to the user with the highest corresponding SINR, i.e. $\max _{1 \leq k \leq K} \mathrm{SINR}_{k, m}$. If we perform the above scheduling, the 
throughput for large $n$ can be written as [10] ${ }^{2}$

$$
R_{R B F}=M E \log \left(1+\max _{1 \leq k \leq K} \operatorname{SINR}_{k, 1}\right)+o(1)
$$

where the term $o(1)$ accounts for the small probability that user $k$ may be the strongest user for more than one beam $\phi_{m}[10]$.

In the group broadcast scenario, we replace each user with a group of users of size $\alpha_{k} n$ (the group of users interested in the same data). In this case a beam is assigned to each group. In order to maximize the throughput, each beam $m$ is assigned to the group with the best equivalent SINR. The equivalent SINR of each group is the minimum of SINR's of decoding the particular beam among users in the group. Therefore, in the group broadcast problem, random beamforming results in the following max min problem

$R_{R B F}=$
$M E \log \left(1+\max _{k}\left\{\min _{i_{1}} \operatorname{SINR}_{i_{1}}, \ldots, \min _{i_{K}} \operatorname{SINR}_{i_{K}}\right\}\right)+o(1)$

In Example 1, we proved that the minimum SINR scales as

$$
\min _{i} \operatorname{SINR}_{i}=\frac{1}{\frac{1}{\rho}+M-1} \frac{1}{n}
$$

From that, we conclude that the for large number of users, the sum rate is given by

$$
R_{R B F}=M \log \left(1+\frac{1}{\left(\frac{1}{\rho}+M-1\right)} \frac{1}{\alpha n}\right)
$$

or for the equal number of users per group case

$$
R_{R B F}=M \log \left(1+\frac{1}{\left(\frac{1}{\rho}+M-1\right)} \frac{K}{n}\right)+o(1)
$$

\section{B. Time Sharing}

Time sharing can achieve better performance even if we force the groups to have an equal time share

$$
\begin{aligned}
R_{\mathrm{TS}} & \geq \frac{1}{K} \sum_{k=1}^{K} \max _{\operatorname{Tr}\left(B_{k}\right)=P} \min _{B_{k} \geq 0} \log \operatorname{det}\left(1+\left\|h_{i_{i_{k}}}\right\|_{B_{k}}^{2}\right) \\
& =\frac{1}{K} \sum_{k=1}^{K} \log \left(1+\max _{\operatorname{Tr}\left(B_{k}\right)=P} \min _{h_{i_{k}}}\left\|h_{i_{k}}\right\|_{B_{k}}^{2}\right)
\end{aligned}
$$

We now relax the problem further by setting $B_{k}=\frac{P}{M} I$, from which we conclude that

$$
R_{\mathrm{TS}} \geq \log \left(1+\frac{P}{M} C_{M} \frac{1}{(\alpha n)^{\frac{1}{M}}}\right)
$$

or using the approximation $\log (1+x)=x+\boldsymbol{O}\left(x^{2}\right)$, and assuming equal number of users

$$
R_{\mathrm{TS}} \geq \frac{P}{M} C_{M} \frac{K^{\frac{1}{M}}}{n^{\frac{1}{M}}}+\boldsymbol{O}\left(\frac{1}{n^{\frac{2}{M}}}\right)
$$

Just like the upper bound, correlation results in a hit $\operatorname{det}(R)^{\frac{1}{M}}$ on the lower bound

$$
R_{\mathrm{TS}, \text { corr }} \geq \frac{P}{M} C_{M} \operatorname{det}(R)^{\frac{1}{M}} \frac{K^{\frac{1}{M}}}{n^{\frac{1}{M}}}+\boldsymbol{O}\left(\frac{1}{n^{\frac{2}{M}}}\right)
$$

\footnotetext{
${ }^{2}$ It turns out that all the beams behave equally. So the sum-rate is simply $M$ times the rate guaranteed by $\phi_{1}$ or any beam for that matter. As such, we drop the beam dependence for notational convenience.
}

\section{Treating Interference as Noise}

The other extreme would be to allow all groups to be served simultaneously. Each group would then ignore signals that are meant for the other groups, treating them as additive noise. The rate that the 1st group achieves with this strategy would be

$$
R_{1}=\min _{i_{1}} \log \left(\frac{h_{i_{1}}^{*} B_{1} h_{i_{1}}}{1+h_{i_{1}}^{*} \sum_{k=2}^{K} B_{k} h_{i_{1}}}\right)
$$

By choosing the $B_{k}$ 's properly, we can maximize the sum rate $\sum_{k} R_{k}$. Now, relax the problem further by assuming equal isotropic covariances for all user groups, i.e. set $B_{k}=\frac{1}{K} \frac{P}{M} I$, then we can show that

$$
\begin{aligned}
R_{1} & =\log \min _{i_{1}} \frac{\frac{1}{K} \frac{P}{M}\left\|h_{i_{1}}\right\|^{2}}{1+\frac{K-1}{K} \frac{P}{M}\left\|h_{i_{1}}\right\|^{2}} \\
& =\frac{1}{K} \frac{P}{M} C_{M} \frac{K^{\frac{1}{M}}}{n^{\frac{1}{M}}}+\boldsymbol{O}\left(\frac{1}{n^{\frac{2}{M}}}\right)
\end{aligned}
$$

Thus, the sum rate for $K$ such user groups is upper bounded according to

$$
R_{\text {Interf }} \geq \frac{P}{M} C_{M} \frac{K^{\frac{1}{M}}}{n^{\frac{1}{M}}}+\boldsymbol{O}\left(\frac{1}{n^{\frac{2}{M}}}\right)
$$

Correlation will again introduce a hit $\operatorname{det}(R)^{\frac{1}{M}}$ on the lower bound

$$
R_{\text {Interf, corr }} \geq \operatorname{det}(R)^{\frac{1}{M}} \frac{P}{M} C_{M} \frac{K^{\frac{1}{M}}}{n^{\frac{1}{M}}}+\boldsymbol{O}\left(\frac{1}{n^{\frac{2}{M}}}\right)
$$

We summarize the findings of sections IV and $\mathrm{V}$ by the following theorem.

Theorem 2: Consider the following group broadcast scenario: A base station with $M$ antennas is to broadcast to $K$ groups of $\frac{n}{K}$ users each, under a total power constraint $P$. Assume also that the users' channels are iid Gaussian. Then the sum-rate capacity behaves like

$$
C=\gamma P C_{M} \frac{K \frac{1}{M}}{n \frac{1}{M}}+\boldsymbol{O}\left(\frac{1}{n \frac{2}{M}}\right)
$$

where $\frac{1}{M} \leq \gamma \leq 1$. When the users' channels exhibit correlation $R$, the capacity scales as

$$
C_{\mathrm{corr}}=\gamma \operatorname{det}(R)^{\frac{1}{M}} P C_{M} \frac{K^{\frac{1}{M}}}{n^{\frac{1}{M}}}+\boldsymbol{O}\left(\frac{1}{n^{\frac{2}{M}}}\right)
$$

These are unfortunate results as they show that the sumrate decreases with the number of users. To counter this, we increase the resources (i.e., number of antennas $M$ ). In the rest of this paper, we study the scaling of group broadcast capacity with the number of antennas when 1) $n$ is fixed, 2) $\frac{M}{n}=\beta$ and 3) $M=\log n$. To simplify the exposition, we will only consider the equal number of users case, i.e. we set $\alpha_{k}=\frac{1}{K} \forall k$.

\section{SCALING WITH $M, n$ FIXED}

In this section, we study the scaling of the group broadcast capacity for large number of antennas $M$ and fixed number of users $n$. We take the upper bound to be $K$ times the maximum rate that a single group achieves

$$
\begin{aligned}
C & \leq K \max _{B \geq 0} \operatorname{Tr}(B) \leq P \\
& \leq K \log \left(1+\min _{i} P\left\|h_{i}\right\|^{2}\right)
\end{aligned}
$$


where the second line follows from the fact that $B \leq P I$. By the law of large numbers we have that $\lim _{M \rightarrow \infty} \frac{\left\|\bar{h}_{i}\right\|^{2}}{M}=$ 1 w.p.1. Since the minimization is taken over a fixed number of users, we conclude that $\lim _{M \rightarrow \infty} \min _{i} \frac{\left\|h_{i}\right\|^{2}}{M}=1$ w.p.1 and

$$
C \leq K \log (1+P M) \text { w.p. } 1
$$

To obtain a lower bound, we use time sharing

$$
C \geq \log \left(1+\max _{B \geq 0} \min _{\operatorname{Tr}(B) \leq P}\left\|h_{i}\right\|_{B}^{2}\right)
$$

To proceed, we need to find a lower bound for $\max _{B \geq 0} \operatorname{Tr}(B) \leq P \min _{i}\left\|h_{i}\right\|_{B}^{2}$ (the lower bound $\min _{i} \frac{P}{M}\left\|h_{i}\right\|^{2}$ is too loose). In Appendix A, we show that for every $h_{i}$ in the set $\left\{h_{1}, h_{2}, \ldots, h_{\frac{n}{K}}\right\}$

$$
\max _{B \geq 0 \operatorname{Tr}(B) \leq P}\left\|h_{i}\right\|_{B}^{2} \geq \frac{P}{\min \left\{M, \frac{n}{K}\right\}}\left\|h_{i}\right\|^{2}
$$

from which we conclude that

$$
\max _{B \geq 0} \min _{\operatorname{Tr}(B) \leq P}\left\|h_{i}\right\|_{B}^{2} \geq \frac{P}{\min \left\{M, \frac{n}{K}\right\}} \min _{i}\left\|h_{i}\right\|^{2}
$$

Now, for fixed $n$ and large $M$, we have by the law of large numbers $\lim _{M \rightarrow \infty} \min _{i} \frac{\left\|h_{i}\right\|^{2}}{M}=1$ and we can write $\max _{B \geq 0} \operatorname{Tr}(B) \leq P \min _{i}\left\|h_{i}\right\|_{B}^{2} \geq P \frac{K}{n} M$. This yields the following lower bound on the sum rate

$$
C \geq \log \left(1+P \frac{K}{n} M\right) \text { w.p.1 }
$$

We thus have the following theorem.

Theorem 3: Consider the setting of Theorem 2 and assume that the total number of users $n$ is kept fixed. Then as the number of antennas grows to infinity, the group broadcast capacity satisfies the following bounds with probability one

$$
\log \left(1+\frac{K}{n} P M\right) \leq C \leq K \log (1+P M)
$$

\section{SCALING WITH $M$ AND $n, \frac{M}{n}=\beta$}

Here we consider the scaling of the upper and lower bounds when both the number of users and antennas grow to infinity while their ratio remains constant $\frac{M}{n}=\beta$. We rely on time sharing to obtain a lower bound

$$
\begin{aligned}
& C \geq K \max _{B \geq 0} \frac{1}{\operatorname{Tr}(B) \leq P} \log \left(1+\min _{i} h_{i}^{*} B h_{i}\right) \\
& C \geq \log \left(1+P \min _{i} \frac{\left\|h_{i}\right\|^{2}}{M}\right)
\end{aligned}
$$

where we have relaxed the problem by setting $B=\frac{P}{M} I$. We now need to lower bound $\min _{i} \frac{\left\|h_{i}\right\|^{2}}{M}$ as $M, n \rightarrow \infty$. To this end, define the matrix

$$
H_{i}=\left[\begin{array}{llll}
h_{1} & h_{2} & \cdots & h_{\frac{n}{K}}
\end{array}\right]
$$

and note that

$$
\operatorname{diag}\left(H_{i}^{*} H_{i}\right)=\left[\begin{array}{llll}
\left\|h_{1}\right\|^{2} & \left\|h_{2}\right\|^{2} & \cdots & \left\|h_{\frac{n}{K}}\right\|^{2}
\end{array}\right]^{T}
$$

Note also that

$\lambda_{\min }\left(H_{i}^{*} H_{i}\right) \leq \min _{i}\left\|h_{i}\right\|^{2} \leq \lambda_{\max }\left(H_{i}^{*} H_{i}\right) \leq \max _{i}\left\|h_{i}\right\|^{2}$

Moreover as $n, M \rightarrow \infty$ with $\frac{M}{n}=\beta$ (so that $\frac{M}{n / K}=K \beta$ ) the eigenvalues of $\frac{H_{i}^{*} H_{i}}{M}$ become uniformly distributed in the range $\left[(1-\sqrt{K \beta})^{2},(1+\sqrt{K \beta})^{2}\right]$ as shown in [21], [22]. We can thus write

$$
(1-\sqrt{K \beta})^{2} \leq \lim _{n, M \rightarrow \infty} \min _{i} \frac{\left\|h_{i}\right\|^{2}}{M} \leq(1+\sqrt{K \beta})^{2}
$$

Combining this with the time sharing bound (25) yields the desired lower bound on the sum-rate capacity

$$
C \geq \log \left(1+P(1-\sqrt{K \beta})^{2}\right)
$$

To obtain an upper bound, we start with the bound

$$
C \leq K \log \left(1+\max _{B \geq 0} \min _{\operatorname{Tr}(B) \leq P}\left\|h_{i}\right\|_{B}^{2}\right)
$$

We now turn our attention to upper bound the log argument (the bound $\max _{B \geq 0} \operatorname{Tr}(B) \leq P \min _{i} h_{i}^{*} B h_{i} \leq P \min _{i}\left\|h_{i}\right\|^{2}$ is too loose). To do so, we will use the dual of the max-min problem derived in Appendix B

$$
\begin{aligned}
\max _{B \geq 0} \min _{\operatorname{Tr}(B) \leq P} h_{i}^{*} B h_{i} & =\min _{\beta_{i} \geq 0, \sum \beta_{i}=1} P \lambda_{\max }\left(\sum_{i} \beta_{i} h_{i} h_{i}^{*}\right) \\
& \leq \frac{K}{n} P \lambda_{\max }\left(\sum_{i} h_{i} h_{i}^{*}\right) \\
& =\frac{K}{n} P \lambda_{\max }\left(H_{i}^{*} H_{i}\right)
\end{aligned}
$$

where $H_{i}$ is as defined in (26). Now, as $n, M \rightarrow \infty$ with $\frac{M}{\frac{n}{K}}=$ $K \beta$, the eigenvalues of $\frac{H_{i} H_{i}^{*}}{n}$ will be confined to the range $\left[\left(1-\frac{1}{\sqrt{K \beta}}\right)^{2},\left(1+\frac{1}{\sqrt{K \beta}}\right)^{2}\right]$. We can thus obtain the following upper bound on capacity

$$
C \leq K \log \left(1+P\left(1+\frac{1}{\sqrt{\beta}}\right)^{2}\right)
$$

From (28) and (32), we see that if we allow the number of antennas to grow linearly with the number of users, we can guarantee a constant sum rate and we can state the following theorem.

Theorem 4: Consider the setting of Theorem 2 and assume that the number of antennas $M$ and the number of users $n$ are made large while maintaining a constant ratio $\frac{M}{n}=\beta$. Then the sum-rate group broadcast capacity will be constant with probability one. More precisely, we have

$\log \left(1+P(1-\sqrt{K \beta})^{2}\right) \leq C \leq K \log \left(1+P\left(1+\frac{1}{\sqrt{\beta}}\right)^{2}\right)$

But is it still possible to do so without straining the resources as much? We answer this question in the next section.

VIII. SCALING With $M$ AND $n, M=\log n$

In the first part of the paper, we proved that the group capacity scales as $\gamma \frac{P}{M} C_{M} \frac{K \frac{1}{M}}{n^{\frac{1}{M}}}$. Now for large $M$, it is easy to prove that $C_{M} \simeq M$ and the sum-rate scales as $\gamma \frac{P}{n \frac{1}{M}}$. Thus, to guarantee a constant rate, we need to set $M=\stackrel{n}{=} \log n$. To prove this rigorously, we first study the behavior of $\min _{i} \frac{\left\|h_{i}\right\|^{2}}{M}$ for large $n$ (and $M$ ). We use the Chernoff bound in Appendix $\mathrm{C}$ to show that

$$
\lim _{M=\log n, n \rightarrow \infty} P\left(\min _{i} \frac{\left\|h_{i}\right\|^{2}}{M} \in\left[1-\epsilon_{l}, 1\right]\right)=1
$$

where $\epsilon_{l} \simeq .8414$, i.e. for $M=\log n$ and for large $n$, $\min _{i} \frac{\left\|h_{i}\right\|^{2}}{M}$ is a constant. 


\section{A. Lower Bound for Sum-rate Capacity ( $M=\log n)$}

We are now ready to derive the lower bound for the sum rate capacity which we obtain through time sharing. Specifically, we have

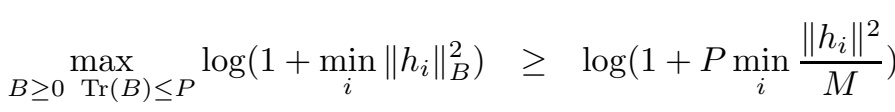

which follows by setting $B=\frac{P}{M} I$. Or with $M=\log n$ and as $n \rightarrow \infty$

$$
C \geq \log (1+P \mathcal{H})
$$

where $\mathcal{H} \in\left[1-\epsilon_{l}, 1\right]$. This lower bound shows that a growth of $M=\log n$ will guarantee a constant capacity because for $M=\beta n$ the sum rate is upper bounded by a constant. This is summarized in the following theorem.

Theorem 5: Consider the setting of Theorem 2 and assume that the number of antennas $M$ and the number of users $n$ are made large while maintaining the relationship $M=\log n$. Then the sum-capacity in such a system scales like a constant and therefore, a constant capacity per user (stream) is achievable in the system.

Theorem 5 suggests that if the number of antennas scale logarithmically with the number of user, i.e. $M=\log n$, constant capacity per user is achievable. In the following theorem we show that to obtain a constant rate per user in the system the number of antennas should at least behave like $(\log n)^{\frac{1}{2}-\epsilon(n)}$ for a vanishing function $\epsilon(n)$.

Theorem 6: Consider the setting of Theorem 2 and assume that the number of users $n$ are made large. Then in order to have a sum-capacity that scales like a constant and therefore, a constant capacity per user (stream) is guaranteed, the number of transmit antennas, $M$, should grow faster than $(\log n)^{\frac{1}{2}-\epsilon(n)}$, where $\epsilon(n)=\frac{\log \log \log n}{\log \log n}$.

Proof: To obtain a constant capacity per user, the multicast capacity of each group should be at least constant. Consider one of the groups with $\alpha n$ users. Then the multicast capacity is given by

$$
\log \left(1+\max _{B \geq 0, \operatorname{Tr} B \leq P} \min _{i} h_{i}^{*} B h_{i}\right)
$$

Therefore, $X=\max _{B \geq 0, \operatorname{Tr} B \leq P} \min _{i}\left\|h_{i}\right\|_{B}$ should be constant as we increase $n$. In Appendix B, we upper bound the above max-min problem by

$$
X \leq P \lambda_{\max }\left(\sum_{i} \beta_{i} h_{i} h_{i}^{*}\right)
$$

for any choice of $\beta_{i} \geq 0$ such that $\sum_{i} \beta_{i}=1$. Now suppose we partition the $\alpha n$ users into sets of size $M$. Then we have $S=\frac{\alpha n}{M}$ sets of users. We can obtain an upper bound by considering a uniform choice $\beta_{i}=\frac{1}{M}$ for all the users in a particular set $s$ and set $\beta_{i}$ to zero for users in the remaining $S-1$ sets. For this choice of $\beta_{i}$, (36) is valid, and we have

$$
X \leq \frac{P}{M} \lambda_{\max }\left(H_{s} H_{s}^{*}\right)
$$

where $H_{s}$ is the channel matrix between the transmitter and the users in the set $s$ of our partition. Since this is true for each of the $S$ sets, we can write

$$
X \leq \frac{P}{M} \min \left(\lambda_{\max }\left(H_{1} H_{1}^{*}\right), \ldots, \lambda_{\max }\left(H_{S} H_{S}^{*}\right)\right)
$$

Note that $H_{s}$ for different $s$ are i.i.d. Hence, we have bounded $X$ by the minimum of $S=\frac{\alpha n}{M}$ i.i.d random variables. In order for the sum-capacity to scale at least like a constant with $n$, the right side of (37) should scale like a constant as well. Let us denote the cumulative distribution function (CDF) of $\lambda_{\max }\left(H H^{*}\right)$ by $F_{W}(\cdot)$, where $H$ is a Gaussian $M \times M$ random matrix. Then $H H^{*}$ is a Wishart Matrix [22], and we can write

$$
\operatorname{Pr}\left(X \geq \frac{1}{P \log M}\right) \leq\left(1-F_{W}\left(\frac{M}{\log M}\right)\right)^{S} .
$$

If $X$ behaves like constant then the above probability should approach one as $n$ tends to infinity. Therefore we should have

$$
\left(1-F_{W}\left(\frac{M}{\log M}\right)\right)^{S} \rightarrow 1
$$

Using the large deviation result of [22] we know that $F_{W}(t)$ behaves like $\exp \left(-M^{2} \Phi_{-}\left(\frac{4 M-t}{M} ; 1\right)\right)$ for $t=O(M)$, where $\Phi_{-}(x ; 1)$ is defined by

$$
\Phi_{-}(x ; 1)=\left(2 \log 2-\log (4-x)-\frac{x}{4}-\frac{x^{2}}{32}\right) u(x)
$$

and where $u(x)$ is the step function. Applying the above large deviation bound to our case yields

$$
F_{W}\left(\frac{M}{\log M}\right) \approx \exp \left(-M^{2} \log \log M\right)
$$

With this in mind, we conclude that for (38) to hold, we should have that for large $n, F_{W}\left(\frac{M}{\log M}\right) \cdot S \rightarrow 0$. Alternatively, we require that

$$
\log S-M^{2} \log \log M \leq 0 .
$$

Substituting $S=\frac{\alpha n}{M}$ in the above inequality we get

$$
\log n \leq\left(M^{2}+1\right) \log \log M(1+o(1))
$$

This gives an upper bound on the maximum number of supportable users, $n$. It can be verified that for $M$ scaling like $(\log n)^{\left(\frac{1}{2}-\epsilon(n)\right)}$ where $\epsilon(n)=\frac{\log \log \log n}{\log \log n}$ this inequality is not satisfied. Therefore, the number of antennas should grow faster than $(\log n)^{\left(\frac{1}{2}-\epsilon(n)\right)}$ with $n$ to guarantee a constant capacity per stream.

\section{CONCLUSION}

In this paper we considered the problem of multi-group broadcast channels. As its name suggests, in group broadcast there are different groups of user in the system, each group of which is interested in common information. This problem can be a good model for digital multimedia broadcasting in wireless networks that is receiving increasing attention in recent years. We specifically studied the scaling of multigroup broadcast for large number of users. We obtained upper and lower bounds for the sum-rate capacity in the large number of users regime. We showed that the sum rate capacity scales as $\gamma P \frac{C_{M}}{n \frac{1}{1}}$. We also quantified the effect of spatial correlation on the sum-rate capacity which manifests itself as a hit of $\operatorname{det}(R)^{\frac{1}{M}}$ on the SNR. This is an unfortunate result as it shows that the capacity decreases with the number of users. To go around this, we studied the scaling of the group broadcast capacity with the number of users and antennas. Specifically, two such regimes were studied 1) when the number of antennas is linear in the number of users and 2) 
when the number of antennas is logarithmic in the number of users. Both of these regimes guarantee a constant rate per user but the logarithmic rate is more tight as we also proved that the number of antennas should at least behave like $(\log n)^{\left(\frac{1}{2}-\epsilon\right)}$ for a constant rate to be maintained.

Some extensions of this work would be to evaluate scaling of group broadcast in the presence of multiple antennas at the users' side and to study the scaling in the wideband case. Another extension would be to study the scaling in the large SNR regime to evaluate the group-broadcast multiplexing gain.

\section{Appendix A}

Consider the set $\mathcal{G}=\left\{h_{1}, h_{2}, \ldots, h_{n}\right\}$. We would like to To prove that for every $h_{i} \in \mathcal{G}$

$$
\max _{B \geq 0 \operatorname{Tr}(B) \leq P} h_{i}^{*} B h_{i} \geq \frac{P}{\min \{M, n\}}\left\|h_{i}\right\|^{2}
$$

Now for $B^{\prime}=\frac{P}{M} I$, we have

$$
\begin{aligned}
\max _{B \geq 0 \operatorname{Tr}(B) \leq P} h_{i}^{*} B h_{i} & \geq h_{i}^{*} B^{\prime} h_{i} \\
& =\frac{P}{M}\left\|h_{i}\right\|^{2}
\end{aligned}
$$

which proves part of (39). Now for $n \leq M$, let $\mathcal{A}=$ $\left\{a_{1}, a_{2}, \ldots, a_{l}\right\}$ be an orthonormal basis for the space spanned by the set $\mathcal{G}$. Then $l \leq n \leq M$. Define the matrix $B^{\prime}$ this time as

$$
B^{\prime}=\frac{P}{n} A A^{*}
$$

where

$$
A=\left[\begin{array}{lllll}
a_{1} & a_{2} & \cdots & a_{l} & O_{M \times M-l}
\end{array}\right]
$$

then $B^{\prime} \geq 0$ and $\operatorname{Tr}\left(B^{\prime}\right)=\frac{l P}{n} \leq P$. Moreover, since each $h_{i}$ is spanned by $\mathcal{A}$, it is easy to see that

$$
h_{i}^{*} B^{\prime} h_{i}=\frac{P}{n}\left\|h_{i}\right\|^{2}
$$

so that

$$
\max _{B \geq 0 \operatorname{Tr}(B) \leq P} h_{i}^{*} B h_{i} \geq \frac{P}{n}\left\|h_{i}\right\|^{2}
$$

Combining (40) and (42) yields the desired result.

\section{APPENDIX B}

To obtain the dual form of the max-min problem of (29), we introduce the dual variables and write the optimization problem in the following form

$$
\begin{aligned}
& \max _{B \geq 0, \operatorname{Tr}(B) \leq P} \min _{i} h_{i}^{*} B h_{i}=\max _{\substack{B \geq 0, t: \\
\operatorname{Tr}(B) \leq P, t \leq h_{i} B h_{i}^{*} \forall i}} t \\
& =\min _{\gamma, \beta_{i} \geq 0: \sum_{i} \beta_{i}=1,} \gamma P \\
& =\min _{\beta_{i} \geq 0: \sum_{i} \beta_{i}=1} \lambda_{\max }\left(\sum_{i} \beta_{i} h_{i} h_{i}^{*}\right)
\end{aligned}
$$

where the third line follows by first minimizing the cost over $\gamma$. Since $\gamma I \geq \sum_{i} \beta_{i} h_{i} h_{i}^{*}$, it readily follows that $\gamma \geq \lambda_{\max }\left(\sum_{i} \beta_{i} h_{i} h_{i}^{*}\right)$, i.e. $\gamma$ is greater equal to the maximum eigenvalue of matrix $\sum_{i} \beta_{i} h_{i} h_{i}^{*}$.

\section{APPENDIX C}

Let $Y=\frac{\left\|h_{i}\right\|^{2}}{M}$, and define $g(Y)$ by

$$
g(Y)= \begin{cases}1 & \text { if } Y \leq 1-\epsilon \\ 0 & \text { if } Y>1-\epsilon\end{cases}
$$

Then for $\nu \geq 0$

$$
g(Y) \leq e^{-\nu(Y-(1-\epsilon))}
$$

which yields the Chernoff bound

$$
E[g(Y)]=P(Y \leq 1-\epsilon) \leq e^{\nu(1-\epsilon)} E\left[e^{-\nu Y}\right]
$$

or

$$
P\left(\frac{\left\|h_{i}\right\|^{2}}{M} \leq 1-\epsilon\right)=e^{\nu(1-\epsilon)} \frac{1}{\left(1+\frac{\nu}{M}\right)^{M}}
$$

Now we can tighten the upper bound by choosing the optimum $\nu$, which, upon setting the first derivative to zero, turns out to be

$$
\nu=M \frac{\epsilon}{1-\epsilon}>0
$$

and the bound reads

$$
\begin{aligned}
P\left(\frac{\left\|h_{i}\right\|^{2}}{M} \leq 1-\epsilon\right) & \leq e^{M \epsilon}(1-\epsilon)^{M} \\
& =e^{M(\epsilon+\log (1-\epsilon))}
\end{aligned}
$$

We can use this to bound the probability $P\left(\min _{i} \frac{\left\|h_{i}\right\|^{2}}{M} \leq\right.$ $1-\epsilon)$

$$
\begin{aligned}
P\left(\min _{i} \frac{\left\|h_{i}\right\|^{2}}{M} \leq 1-\epsilon\right) & =1-\left(1-P\left(\frac{\left\|h_{i}\right\|^{2}}{M} \leq 1-\epsilon\right)\right)^{n} \\
& \leq 1-\left(1-e^{M(\epsilon+\log (1-\epsilon))}\right)^{n} \\
& =1-\left(1-n^{\epsilon+\log (1-\epsilon)}\right)^{n}
\end{aligned}
$$

where the last line follows from the fact that $M=\log n$. For the above probability to vanish as $n$ grows, we require that

$$
\epsilon+\log (1-\epsilon)<-1
$$

Let $\epsilon_{l}$ be the infimum of the set $\{\epsilon: \epsilon+\log (1-\epsilon)<-1\}$, (i.e. $\epsilon_{l}$ satisfies $\epsilon_{l}+\log \left(1-\epsilon_{l}\right)=-1$ or $\epsilon_{l} \simeq$.8414). Then,

$$
\lim _{n \rightarrow \infty} P\left(\min _{i} \frac{\left\|h_{i}\right\|^{2}}{M} \geq 1-\epsilon_{l}\right)=1
$$

Now let's obtain an upper bound for $\min _{i} \frac{\left\|h_{i}\right\|^{2}}{M}$. Employing Chernoff bound again, it is easy to show that for $\nu \geq 0$

$$
\begin{aligned}
P\left(\frac{\left\|h_{i}\right\|^{2}}{M} \geq 1+\epsilon\right) & \leq e^{-\nu(1+\epsilon)} E\left[e^{\nu \frac{\left\|h_{i}\right\|^{2}}{M}}\right] \\
& =e^{-\nu(1+\epsilon)} \frac{1}{\left(1-\frac{\nu}{M}\right)^{M}}
\end{aligned}
$$

Moreover, the upper bound is tightest for $\nu=M \frac{\epsilon}{1+\epsilon}$ We thus have

$$
\begin{aligned}
P\left(\frac{\left\|h_{i}\right\|^{2}}{M} \geq 1+\epsilon\right) & \leq e^{-M \epsilon}(1+\epsilon)^{M} \\
& =e^{M(-\epsilon+\log (1+\epsilon))}
\end{aligned}
$$

or

$$
P\left(\min _{h_{i}} \frac{\left\|h_{i}\right\|^{2}}{M} \geq 1+\epsilon\right) \leq\left(n^{(-\epsilon+\log (1+\epsilon))}\right)^{n}
$$


where we used the fact that $n=\log M$. This probability vanishes provided that $-\epsilon+\log (1+\epsilon)<0$ and the infimum for which this is true is $\epsilon_{u}=0$. We can thus write

$$
\lim _{n \rightarrow \infty} P\left(\min _{i} \frac{\left\|h_{i}\right\|^{2}}{M} \leq 1\right)=1
$$

From (44) and (47), we see that

$$
\lim _{n \rightarrow \infty} P\left(\min _{i} \frac{\left\|h_{i}\right\|^{2}}{M} \in\left[1-\epsilon_{l}, 1\right]\right)=1
$$

\section{REFERENCES}

[1] B. Hochwald, T. Marzetta, and V. Tarokh, "Multi-antenna channelhardening and its implications for rate feedback and scheduling," IEEE Trans. Inform., vol. 50, no. 9, pp. 1893-1909, Sept. 2004.

[2] E. Telatar, "Capacity of multi-antenna Gaussian channel," European Trans. Telecommun., vol. 10, pp. 585-595, Nov. 1999.

[3] G. J. Foschini and M. J. Gans, "On limits of wireless communications in a fading environment when using multiple antennas," Wireless Personal Commun., vol. 6, pp. 311-335, Mar. 1998.

[4] A. Goldsmith, S. A. Jafar, N. Jindal, and S. Vishwanath, "Capacity limits of MIMO channels," IEEE J. Select. Areas. Commun., vol. 21, no. 5, pp. 684-702, June 2003.

[5] S. Vishwanath, N. Jindal, and A. Goldsmith, "Duality, achievable rates and sum rate capacity of Gaussian MIMO broadcast channel," IEEE Trans. Inform. Theory, vol. 49, no. 10, pp. 2658-2668, 2003.

[6] P. Viswanath and D. N. Tse, "Sum capacity of the vector Gaussian broadcast channel and downlink-uplink duality," IEEE Trans. Inform. Theory, vol. 49, no. 8, pp. 1912-1921, Aug. 2003.

[7] H. Weingarten, Y. Steinberg, and S. Shamai, "The capacity region of the gaussian MIMO broadcast channel," in Proc. International Symposium on Information Theory (ISIT), 2004.

[8] Q. H. Spencer, C. B. Peel, A. L. Swindlehurst, and M. Haardt, "An introduction to the multi-user MIMO downlink," IEEE Commun. Mag., vol. 42 , no. 10 , pp. $60-67$, Oct. 2004.

[9] M. Sharif and B. Hassibi, "Scaling laws of sum rate using timesharing, DPC, and beamforming for MIMO broadcast channels," in Proc. International Symposium on Information Theory (ISIT), 2004.

[10] M. Sharif and B. Hassibi "On the capacity of MIMO broadcast channel with partial side information," IEEE Trans. Inform. Theory, vol. 51, no. 2, Feb. 2005

[11] P. Viswanath, D. N. Tse, and R. Laroia, "Opportunistic beamforming using dump antennas," IEEE Trans. Inform. Theory, vol. 48, no. 6, pp. 1277-1294, June 2002.

[12] T. Yoo and A. Goldsmith, "On the Optimality of multi-antenna broadcast scheduling using zero-forcing beamforming," IEEE J. Select. Areas. Commun., vol. 24, no. 3, pp. 528-541, Mar. 2006.

[13] G. A. Jones, J. M. Defilippis, H. Hoffmann, and E. A. Williams, "Digital television station and network implementation," Proc. IEEE, vol. 94, no. 1, pp. 22-36, Jan. 2006.

[14] U. Reimers, "Digital video broadcasting," IEEE Commun. Mag., vol. 36, no. 6, pp. 104-110, June 1998

[15] S. Pekowsky and A. Andorfer, "Multimedia data broadcasting strategies," IEEE Commun. Mag., vol. 39, no. 4, pp. 138-145, Apr. 2001.

[16] A. Khisti, U. Erez, A. Lapidoth, G. Wornell, "Carbon copying onto dirty paper," submitted to IEEE Trans. Inform. Theory.

[17] Y. Steinberg and S. Shamai, "Achievable rates for the broadcast channel with states known at the transmitter," in Proc. International Symposium on Information Theory (ISIT), pp. 2184-2188, Sept. 2005.

[18] A. Khitsi, "Coding techniques for multicasting," Master's thesis, MIT, Cambridge, MA, 2004.

[19] N. Jindal and Z.-Q. Luo, "Capacity limits of multiple antenna multicast," in Proc. International Symposium on Information Theory (ISIT), Seattle, USA, July 2006.

[20] S. Vishwanath, N. Jindal, and A. Goldsmith, "Duality, achievable rates and sum rate capacity of Gaussian MIMO broadcast channel," IEEE Trans. Inform. Theory, vol. 49, no. 10, pp. 26582668, 2003.
[21] V. A. Marcenko and L. A. Pastur, "Distribution of eigenvalues for some sets of random matrices," Math USSR Sbornik, vol. 1, pp. 457-483, 1967.

[22] P. Vivo, S. N. Majumdar and O. Bohigas, "Large deviations of the maximum eigenvalue in Wishart random matrices," J. Phys. A: Math. Theor, vol. 40, pp. 4317-4337, 2007.

Tareq Y. Al-Naffouri obtained his M.S. degree in electrical engineering from Georgia Institute of Technology, Atlanta, in 1998, and his Ph.D. in Electrical Engineering from Stanford University, CA in 2004. He was a visiting scholar at California Institute of Technology, Pasadena, CA, in 2005 and during the summer of 2006, and a Fulbright scholar at the University of Southern California in 2008. He has held internship positions at NEC Research Labs, Tokyo, Japan in 1998, Adaptive Systems Lab, University of California at Los Angeles in 1999, National Semiconductor, Santa Clara, CA, in 2001 and 2002, and Beceem Communications Santa Clara, CA, in 2004. Dr Al- Naffouri is currently an Assistant Professor at the Electrical Engineering Department, King Fahd University of Petroleum and Minerals, Saudi Arabia. His research interests lie in the areas of adaptive and statistical signal processing and their applications to wireless communications and in multiuser information theory. $\mathrm{He}$ has over 50 publications in Journal and Conference Proceedings. Dr AlNaffouri is the recipient of a 2001 best student paper award at the IEEEEURASIP Workshop on Nonlinear Signal and Image Processing (NSIP) 2001 for his work on adaptive filtering analysis.

Amir F. Dana (S '98) was born in Tehran, Iran, in 1979. He received the B.S. degree in electrical engineering from the Sharif University of Technology, Tehran, Iran, in 2001, and the M.S. and Ph.D. degrees in electrical engineering from the California Institute of Technology, Pasadena, in 2002 and 2006, respectively. He is currently with Qualcomm, Inc., San Diego, CA. His research interests are in the areas of information theory, and wireless communications. In particular, he has been working on power efficiency of sensor and ad hoc networks, network coding for wireless networks, and capacity of broadcast channels.

Babak Hassibi was born in Tehran, Iran, in 1967. He received the B.S degree from the University of Tehran in 1989, and the M.S. and Ph.D. degrees from Stanford University in 1993 and 1996, respectively, all in electrical engineering. From October 1996 to October 1998 he was a research associate at the Information Systems Laboratory, Stanford University, and from November 1998 to December 2000 he was a Member of the Technical Staff in the Mathematical Sciences Research Center at Bell Laboratories, Murray Hill, NJ. Since January 2001 he has been with the department of electrical engineering at the California Institute of Technology, Pasadena, CA., where he is currently an associate professor. He has also held shorttem appointments at Ricoh California Research Center, the Indian Institute of Science, and Linkoping University, Sweden. His research interests include wireless communications, robust estimation and control, adaptive signal processing and linear algebra. He is the coauthor of the books Indefinite Quadratic Estimation and Control: A Unified Approach to H2 and H1 Theories (New York: SIAM, 1999) and Linear Estimation (Englewood Cliffs, NJ: Prentice Hall, 2000). He is a recipient of an Alborz Foundation Fellowship, the 1999 O. Hugo Schuck best paper award of the American Automatic Control Council, the 2002 National Science Foundation Career Award, the 2002 Okawa Foundation Research Grant for Information and Telecommunications, the 2003 David and Lucille Packard Fellowship for Science and Engineering and the 2003 Presidential Early Career Award for Scientists and Engineers (PECASE). He has been a Guest Editor for the IEEE TRANSACTIONS ON INFORMATION THEORY special issue on space-time transmission, reception, coding and signal processing was an Associate Editor for Communications of the IEEE TRANSACTIONS ON INFORMATION THEORY during 2004-2006, and is currently an Editor for the journal FOUNDATIONS AND TRENDS IN INFORMATION AND COMMUNICATION. 